\title{
Long-Wavelength Neutron Scattering Studies of the Decomposition of Al-Zn Alloys
}

\author{
By C. D. Clark, S. Messoloras, E. W. J. Mitchell and R. J. Stewart \\ J. J. Thomson Physical Laboratory, Whiteknights, Reading RG $62 A F$, England
}

(Received 29 April 1974)

\begin{abstract}
The initial stages of the decomposition sequence of low-zinc-concentration aluminum alloys at room temperature, after being quenched from an annealing temperature in the range 175 to $400^{\circ} \mathrm{C}$ (i.e. from the $\alpha$ phase) are thought to be determined either by a spinodal mechanism or by a nucleation and growth mechanism giving rise to Guinier-Preston zones. In an attempt to resolve which of these mechanisms is operative long-wavelength neutron scattering measurements have been performed on $\mathrm{Al}-\mathrm{Zn}$ alloys containing $1 \cdot 5,12$ and 15 at. \% zinc. These measurements have been made with the small-angle scattering apparatus at the HFR in Grenoble. The results of a study of the stages of the decomposition corresponding to aging times at room temperature between $2 \mathrm{~min}$ and 1 year are discussed. In addition some preliminary results on the dependence of the rate of decomposition upon the annealing temperature are reported.
\end{abstract}

J. Appl. Cryst. (1975). 8, 127

\section{Scattering of X-rays by Correlated Defect Distributions}

BY I. GAAL

Institute for Technical Physics of the Hungarian Academy of Sciences, Budapest, Hungary

(Received 29 April 1974)

It may be essential in certain cases (e.g. non-dilute alloys and dislocations) to take into account the (pair and higher-order) correlations in the defect distribution. To this end the kinematic expression for the total scattered intensity was rewritten into the following form:

$$
\begin{gathered}
I(\mathbf{K}+\mathbf{g})=\sum_{m} \exp (i g A m) \sum_{n} \exp S(\mathbf{K}, n, m) \\
S(\mathbf{K}, n, m)=\sum_{p=1}^{\infty} \frac{1}{p !} \int \ldots \int\left(\begin{array}{l}
p \\
q
\end{array}\right) \prod_{l=1}^{q} \alpha_{1}\left(n, \boldsymbol{\rho}_{l}\right) \prod_{k=q+1}^{p} \alpha_{2}\left(n, \boldsymbol{\rho}_{k}\right) g^{q, p-q}\left(\boldsymbol{\rho}_{1} \ldots \boldsymbol{\rho}_{2}\right) \mathrm{d} \boldsymbol{\rho}_{1} \ldots \mathrm{d} \boldsymbol{\rho}_{p}
\end{gathered}
$$

with

$$
\alpha_{j}=\exp \left\{i \mathbf{K}\left[\mathbf{u}_{j}\left(n+m, \boldsymbol{\rho}_{s}\right)-\mathbf{u}_{j}\left(n_{1} \boldsymbol{\rho}_{s}\right)\right]\right\}-1 \quad(j=1,2)
$$

where $\mathbf{K}$ is the Bravais vector of the reflexion, $\mathbf{u}_{i}(\mathbf{n}, \boldsymbol{\rho})$ denotes the (elastic) displacement field at the lattice point A $n$ caused by the $i$ th kind of single defect (being at lattice point $\rho$ ) and $g^{q, p-q}\left(\rho_{1} \ldots \rho_{p}\right)$ is one of the $p$ th order Ursell-Mayer functions describing the correlation between $q$ defects of $\mathbf{u}_{1}$ type and $p-q$ defects of $\mathbf{u}_{2}$ type. For vanishing $p$ th-order correlations the $s \geq p$ th order Ursell-Mayer functions vanish and for vanishing second-order correlations equation (1) is equivalent to Krivoglaz's equation. For dislocations the second-order correlations are essential; at small $m S$ is proportional to $m^{2} \log \left[B^{2}(|\mathbf{A} m|)^{-2}\right]$, where $B$ is the decay length of the screening-type second-order correlations. $S$ is never proportional to $K^{2}$; there is a finite intercept on the Warren-Averbach plot, but it depends on $m^{2}$ and therefore it cannot be interpreted as an apparent particle size. At large $m S$ is logarithmically divergent for dipole-like screening and it tends to a finite value for quadrupole screening. In the latter case the scattering intensity can be separated into Bragg and diffuse scattering. 